\title{
Influenza Vaccination Documentation Rates During the First Year After Diagnosis of Diffuse Large B Cell Lymphoma
}

\author{
Andres Chang, M.D., Ph.Da,b, Jackelyn B. Payne, M.P.H. ${ }^{a}$, Pamela B. Allen, M.D., M.Sc. ${ }^{a}$, \\ Jean L. Koff, M.D., M.Sc. ${ }^{a}$, Rafi Ahmed, Ph.D. ${ }^{b}$, Christopher R. Flowers, M.D., M.Sc. ${ }^{a}$, and \\ Robert A. Bednarczyk, Ph.D. ${ }^{C}$ \\ aDepartment of Hematology and Medical Oncology, Winship Cancer Institute, Emory University \\ School of Medicine, Atlanta, GA 30322 \\ ${ }^{b}$ Emory Vaccine Center, Department of Microbiology and Immunology, Emory University School \\ of Medicine, Atlanta, GA 30322 \\ 'Hubert Department of Global Health, Rollins School of Public Health, Emory University, Atlanta, \\ GA 30322
}

\begin{abstract}
Introduction: Influenza infection causes significant morbidity and mortality in patients with cancer, and annual influenza vaccination for individuals with cancer is recommended. We sought to examine the documentation rate of influenza vaccine administration, refusal, or counseling in the first year after diagnosis of diffuse large B cell lymphoma (DLBCL) for patients across three hospitals in two healthcare systems.
\end{abstract}

Patients and Methods: Documentation of vaccine administration, refusal, or counseling by physicians, advanced practice providers, or nursing staff during the first period of influenza vaccine availability after diagnosis (August to April) was assessed in medical records of patients diagnosed with DLBCL between February 2015 and October 2017 who presented to Emory St. Joseph Hospital (community hospital), Winship Cancer Institute of Emory University (academic medical center), or Grady Memorial Hospital (county hospital).

Results: Of the 57\% (61/107) of newly-diagnosed DLBCL patients who had vaccine-related documentation, $43 \%$ refused vaccination. Counseling was not documented for any patient.

\footnotetext{
Corresponding Author: Andres Chang, MD, PhD, Department of Hematology and Medical Oncology, Winship Cancer Institute of Emory University, Emory University School of Medicine, 1365 Clifton Road NE, C5001, Atlanta, GA 30322, Phone: 404-778-1900, andres.chang@emory.edu.

Publisher's Disclaimer: This is a PDF file of an unedited manuscript that has been accepted for publication. As a service to our customers we are providing this early version of the manuscript. The manuscript will undergo copyediting, typesetting, and review of the resulting proof before it is published in its final citable form. Please note that during the production process errors may be discovered which could affect the content, and all legal disclaimers that apply to the journal pertain.

Statement of Conflict of Interest

A.C. is the recipient of the Winship Cancer Institute of Emory University Neil W. And William S. Elkin award and an awardee in the NIH T32 training grant T32CA160040.

R.A. has a patent for the use of PD-1 antagonists for the treatment of infections and tumors. This conflict is monitored by Emory University and is outside the scope of the submitted work.

C.R.F. receives research grants from Celgene and other research funding from AbbVie, Acerta, Gilead, Janssen, Millennium, Infinity, Pharmacyclics, TG Therapeutics, and personal fees from Bayer and Celgene outside of the scope of the submitted work.

The remaining authors have no relevant conflicts of interest to declare.
} 
Inpatient nursing performed $75 \%$ of all documentation. Primary oncologists documented vaccination in $4 \%$ of all cases.

Conclusion: Despite the limited immunization documentation and high refusal rates observed in this study, the influenza vaccine refusal rate was lower than the average for the United States, the state of Georgia, and the previous studies of patients with cancer. Although routine outpatient vaccination occurs, improvements in screening, strategies for sharing patient vaccine-related information, and counseling of patients who refuse the vaccine are needed. Further work is also needed to determine the effectiveness of influenza vaccination in patients receiving anti-cancer therapy.

\section{Microabstract}

Annual influenza vaccination is recommended for patients with cancer. We assessed documentation on influenza vaccine administration, refusal, or counseling in medical records of 114 patients with a newly-diagnosed lymphoma. Of the $57 \%$ of patients who had vaccine-related documentation, $43 \%$ refused vaccination. Counseling was not documented for any patient. Strategies to improve documentation and counseling are needed to improve patient care.

\section{Keywords}

Flu vaccine; DLBCL; non-Hodgkin lymphoma

\section{Introduction}

Influenza infection is a significant cause of mortality in patients with cancer, with case fatalities surpassing $10 \%{ }^{1}$. It is also a significant cause of morbidity in this population as influenza infection can lead to hospitalizations, infectious complications such as bacterial pneumonia, respiratory failure requiring mechanical ventilation, and deconditioning, many times causing significant delays in cancer treatment. Therefore, guidelines from multiple societies including the American Cancer Society (ACS), the Centers for Disease Control and Prevention $(\mathrm{CDC})^{2}$, and the National Comprehensive Cancer Network $(\mathrm{NCCN})^{3}$ recommend annual influenza vaccination for all individuals with cancer. Because the effectiveness of influenza vaccination can be lower in patients undergoing active cancer treatment, vaccination of family members, caregivers, and healthcare providers with the annual inactivated influenza vaccine is also strongly encouraged ${ }^{4}$.

Despite recommendations from multiple societies, vaccination coverage in the United States, including patients with cancer, is low. Recent data from the U.S. Center for Disease Control and Prevention estimate that influenza vaccination coverage nationwide and in the state of Georgia remains around $40 \%{ }^{5}$. While similar vaccination rates are reported for patients with cancer $^{6,7}$, information about vaccination rates in patients with lymphoma is lacking. We performed a retrospective study to evaluate documentation of influenza vaccination and vaccination rates in patients with newly-diagnosed diffuse large B cell lymphoma (DLBCL) during the first influenza season following diagnosis. 


\section{Patients and Methods}

Medical records were reviewed and manually extracted for 114 patients with a new diagnosis of DLBCL between February 1, 2015 to October 31, 2017 who presented to either Emory St. Joseph Hospital (a community hospital setting), Winship Cancer Institute at Emory University (an academic medical center), or Grady Memorial Hospital (a county hospital) through a protocol approved by the Emory University Institutional Review Board. Documentation of administration, refusal, or discussion regarding influenza vaccination by physicians, advanced practice providers, or nursing staff during the first influenza season (defined as August to April) after diagnosis was assessed on patients who presented at least once during that timeframe. Documentation from all oncology clinic and hospital admission encounters that occurred during the first influenza season after diagnosis were examine for each patient. Plots were generated using GraphPad Prism 7.

\section{Results}

A total of 114 patients with newly diagnosed DLBCL were identified. One patient was excluded from analysis because she was enrolled in hospice prior to influenza season and 6 others were excluded because they did not have any encounters during their first influenza season after diagnosis. The median age at diagnosis was 59 years (range 21-88 years), and $34 \%$ of patients were over 65 years of age at the time of diagnosis. Fifty-three percent of patients were male and the majority were Caucasian. Table 1 shows the demographic and clinical characteristics of patients in the study. Most of the patients in the study were seen at the academic medical center $(66 \%, 71 / 107)$ followed by the community hospital $(23 \%$, $25 / 107)$ and the county hospital $(10 \%, 11 / 107)$ respectively. Influenza vaccination status within 1 year of diagnosis was documented for $57 \%$ (61/107) of patients with a higher rate of documentation of vaccination status in the county hospital and a lower rate in the community hospital (Figure 1A, Table 2). To further define the role of different healthcare providers in documenting vaccination status, we reviewed the role of the providers who provided documentation of vaccination status. Documentation of influenza vaccination status by primary oncologists or advanced practice providers was observed in 4 of the 107 cases (4\% of all cases), accounting for 7\% (4/61) of the patients who had documentation on influenza vaccination (Figure 1B). These 4 patients also had documentation from the outpatient nursing staff who subsequently administered the influenza vaccine. Interestingly, most of the documentation related to influenza vaccination in this cohort was performed by inpatient nursing staff at the time of a hospital admission (Figure 1B). None of these patients had documentation of influenza vaccination status by an inpatient oncologist or advanced practice provider.

In addition to vaccine documentation rates, we sought to examine the vaccination rate among patients with newly-diagnosed DLBCL who were treated in these clinical settings. Among the patients with documentation of influenza vaccination status, the vaccination rate was 57\% (35/61). Similar vaccination rates were observed among the three hospitals (Figure 2 , Table 2). No reason was documented for vaccine refusal, and there was no follow up documentation on vaccine counseling by nursing staff, physicians, or advanced practice providers in any of these admitted patients. 


\section{Discussion}

This report shows that there are deficiencies in the documentation of influenza vaccination status among the 3 different healthcare settings studied, particularly in the outpatient setting. Despite recommendations from multiple societies and all of the practitioners caring for patients in each of these settings on the importance of influenza vaccine for the prevention of influenza infection, a large percentage of individuals with a new diagnosis of DLBCL did not have any documentation of influenza vaccine administration or counseling. This lack of documentation limited our ability to more accurately assess influenza vaccination rates in patients with newly-diagnosed DLBCL at these institutions. Several factors are likely contributing to the large number of patients without documentation of influenza vaccination status. First, the hospitals assessed in this study have an inpatient vaccine screening protocol implemented, but none have a protocol to screen patients with lymphomas in the outpatient setting. Additionally, we were unable to obtain documentation from patients who may have received an influenza vaccine from other providers outside such as primary care physicians, health departments, health fairs, pharmacies, urgent care centers, or other hospitals unless they underwent inpatient vaccine screening. Exceptions included 2 patients who have their primary and oncology care within the county hospital (Figure 1B). Routine outpatient vaccination screening and strategies for sharing and linking patient vaccination status between providers in different healthcare systems at the state and national levels could improve vaccination documentation in patients with lymphoma and provide opportunities to improve compliance. Integrating data from state-wide immunization registry systems like the Georgia Registry of Immunization Transactions and Services (GRITS), into routine medical practice and electronic medical records at Oncology clinics may improve documentation of vaccination and provide additional opportunities to improve counseling and vaccine compliance. Most of the documentation of influenza vaccination status among all three settings was performed by inpatient nursing staff (Figure 1B). It is noteworthy that none of these patients had documentation by inpatient physicians or advanced practice providers stating whether the patient received or refused the influenza vaccine on admission. Similarly, there was no documentation by the nurse performing the screen that a physician or advanced practice provider was informed that the vaccine was refused, and none of the patients who refused the influenza vaccine had documentation on counseling regarding influenza vaccination. This observation calls into question the utility of vaccination screens if physicians and advanced practice providers are not made aware of patients refusing vaccination or do not provide vaccination counseling. The implementation of additional steps to vaccination protocols that alert providers of vaccine refusals and encourage providers to counsel patients on the importance of vaccination may lead to an overall decrease in the vaccination refusal rate observed in this study. Multiple studies have been performed to determine optimal strategies at increasing vaccination rates in different populations. They have tested different intervention strategies including home visits, increasing availability at different locations, incentives and subsidies, implementation of standing orders and other healthcare system-based changes, and reminders to clients and providers. These strategies have led to increases in up to $80 \%$ compared to no intervention with a median cost of $\$ 3.27$ per enrollee and $\$ 50.78$ per additional enrollee vaccinated ${ }^{8}$. These costs are similar to those described in a study conducted by the Community 
Preventive Services Task Force, in which the cost effectiveness of different intervention strategies was also analyzed. In that report, reminder systems to providers and patients were shown to have the lowest implementation cost $^{9}$. New models accounting for healthcare delivery system, provider, and patient-specific factors ${ }^{10}$ should be further explored to increase influenza vaccination rates.

Despite the lack of documentation in a significant number of patients with a new diagnosis of DLBCL, the rates of influenza vaccine refusal observed in this study was lower than the average for the United States, the state of Georgia ${ }^{5}$, and the previously published studies in patients with cancer ${ }^{6,7}$. Of note, O'Halloran, et. al. analyzed data from the 2012-2013 Behavioral Risk Factor Surveillance System to estimate influenza vaccination coverage in adults with high-risk conditions, including cancer. Their report did not assess for differences in vaccination rates between patients with different types of malignancies and did not distinguish individuals receiving active treatment from those who were on surveillance. Loulergue, et. al. prospectively analyzed 112 patients receiving chemotherapy for colorectal, lung, prostate, sarcoma, and urothelial cancers in 2008 but did not include patients with hematological malignancies. Thus, these patients would not have received agents that target specifically immune cells and could lead to a decreased immune response such as the CD20targeted monoclonal antibody rituximab. To our knowledge, this is the first study that provides information specific for newly-diagnosed DLBCL. While the observed rate of vaccine refusal in our cohort was lower than has been observed in the general population ${ }^{5}$, it remains surprisingly high given that patients with lymphomas receive additional education about the risks of immune suppression and infection during and following treatment compared with the general population. These findings highlight the need for further education about the risks of an immunocompromised state, and underscores the need to understand the perception of influenza vaccine perception in patients and their closed relatives to understand the reasons behind vaccine refusal.

There are currently conflicting data on the effectiveness of the seasonal influenza vaccine in patients with lymphoma. Some early studies have reported that patients with lymphoma have a lower response to influenza vaccination after treatment with rituximab ${ }^{11-13}$. While these studies may lead some to believe that influenza vaccination is not useful in patients with a new diagnosis of DLBCL, more recent data show that patients who had been exposed to rituximab can still mount a humoral immune response despite the lack of CD20+ B-cells in the peripheral blood ${ }^{14,15}$. It remains to be seen whether patients receiving active treatment with rituximab-containing regimens can elicit a humoral immune response to the vaccine and whether the effectiveness of vaccination in patients with lymphoma is related to the timing of vaccination in relation to chemoimmunotherapy. Studies are urgently needed to directly assess influenza vaccine efficacy at inducing immunity and preventing influenza infection in lymphoma patients who are actively receiving treatment. Clear evidence of efficacy would further highlight the importance of implementing protocols for influenza vaccination and counseling in the outpatient setting. 


\section{Conclusions}

Our study shows that documentation of influenza vaccination status in patients with newlydiagnosed DLBCL is suboptimal in three clinical settings, most documentation of vaccination was performed by inpatient nursing staff at the time of hospital admission, and no follow-up education was provided to individuals who refused the influenza vaccine. Implementation of outpatient vaccination screening and improved vaccine counseling strategies for patients and caregivers will likely improve documentation status and compliance rate in this patient population, who are already at higher risk of having complications from severe influenza infections. Further work is also needed to determine the effectiveness of influenza vaccination in patients receiving aggressive chemoimmunotherapy to determine the optimal vaccination schedule in this population.

\section{Clinical Practice Points}

This study shows that documentation of influenza vaccination status and the percent of patients who received the seasonal influenza vaccine during the first year of diagnosis of DLBCL are suboptimal in three different healthcare settings and that $>40 \%$ of patients did not have documentation of influenza vaccination status. Although the influenza vaccination rate among patients who had a documented vaccination status was higher than the national average, over $40 \%$ of the patients screened refused the influenza vaccine. Alarmingly, none of these patients received counseling after refusing the vaccine. Additionally, this study shows that routine inpatient vaccination screening protocols can be useful to assess the vaccination status of a patient and suggests that similar protocols implemented in the outpatient setting could provide similar results. However, it is unlikely that implementation of these protocols alone would lead to a significant change in patients' perception of the vaccine unless counseling is incorporated for patients who refuse vaccination. To improve compliance with seasonal influenza vaccination in this vulnerable population, we recommend the implementation of routine vaccination screening protocols in the inpatient and outpatient settings that incorporate lymphoma-specific counseling for individuals who refuse vaccinations.

\section{Acknowledgements}

We would like to thank members of the Winship Cancer Institute Lymphoma Program and the Lymphoma Epidemiology Outcomes Study for identifying patients with a new diagnosis of lymphoma. This work was supported by the Winship Cancer Institute at Emory University T.J. Martell award to R.A., the Nell W. and William S. Elkin Fellowship to A.C.; the National Institutes of Health NCI grant T32CA160040 to which A.C is an awardee and NCI grants U01CA195568 and K24CA208132 to C.F., and NIAID grant K01AI106961 to R.B.

\section{References}

1. Kunisaki KM, Janoff EN. Influenza in immunosuppressed populations: a review of infection frequency, morbidity, mortality, and vaccine responses. Lancet Infect Dis 2009;9:493-504. [PubMed: 19628174]

2. Grohskopf LA, Sokolow LZ, Broder KR, et al. Prevention and Control of Seasonal Influenza with Vaccines: Recommendations of the Advisory Committee on Immunization Practices — United States, 2017-18 Influenza Season. MMWR Recomm Rep 2017;66:1-20. 
3. Baden LR, Swaminathan S, Angarone M, et al. Prevention and Treatment of Cancer-Related Infections, Version 2.2016, NCCN Clinical Practice Guidelines in Oncology. Journal of the National Comprehensive Cancer Network 2016;14:882-913. [PubMed: 27407129]

4. Ward EM, Flowers CR, Gansler T, Omer SB, Bednarczyk RA. The importance of immunization in cancer prevention, treatment, and survivorship. CA Cancer J Clin 2017;67:398-410. [PubMed: 28753241]

5. Santibanez TA, Zhai Y, O'Halloran A, et al. Cumulative monthly influenza vaccination coverage estimates for persons 6 months and older by State, HHS Region, and the United States, National Immunization Survey-Flu (NIS-Flu) and Behavioral Risk Factor Surveillance System (BRFSS), 2016-17 influenza season. FluVaxView 2018.

6. O’Halloran AC, Lu P-j, Williams WW, Bridges CB, Singleton JA. Influenza Vaccination Coverage Among People With High-Risk Conditions in the U.S. American Journal of Preventive Medicine 2016;50:e15-e26. [PubMed: 26238603]

7. Loulergue P, Mir O, Alexandre J, Ropert S, Goldwasser F, Launay O. Low influenza vaccination rate among patients receiving chemotherapy for cancer. Annals of Oncology 2008;19:1658-.

8. Anderson LJ, Shekelle P, Keeler E, et al. The Cost of Interventions to Increase Influenza Vaccination: A Systematic Review. Am J Prev Med 2018;54:299-315. [PubMed: 29362167]

9. Jacob V, Chattopadhyay SK, Hopkins DP, et al. Increasing Coverage of Appropriate Vaccinations: A Community Guide Systematic Economic Review. Am J Prev Med 2016;50:797-808. [PubMed: 26847663]

10. Bednarczyk RA, Chamberlain A, Mathewson K, Salmon DA, Omer SB. Practice-, Provider-, and Patient-level interventions to improve preventive care: Development of the P3 Model. Prev Med Rep 2018;11:131-8. [PubMed: 30003011]

11. Yri OE, Torfoss D, Hungnes $\mathrm{O}$, et al. Rituximab blocks protective serologic response to influenza A (H1N1) 2009 vaccination in lymphoma patients during or within 6 months after treatment. Blood 2011;118:6769-71. [PubMed: 22058114]

12. Monkman K, Mahony J, Lazo-Langner A, Chin-Yee BH, Minuk LA. The pandemic H1N1 influenza vaccine results in low rates of seroconversion for patients with hematological malignancies. Leuk Lymphoma 2011;52:1736-41. [PubMed: 21663502]

13. Bedognetti D, Zoppoli G, Massucco C, et al. Impaired response to influenza vaccine associated with persistent memory B cell depletion in non-Hodgkin's lymphoma patients treated with rituximab-containing regimens. J Immunol 2011;186:6044-55. [PubMed: 21498665]

14. Cho A, Bradley B, Kauffman R, et al. Robust memory responses against influenza vaccination in pemphigus patients previously treated with rituximab. JCI Insight 2017;2.

15. Bedognetti D, Ansaldi F, Zanardi E, et al. Seasonal and pandemic (A/H1N1 2009) MF-59adjuvanted influenza vaccines in complete remission non-Hodgkin lymphoma patients previously treated with rituximab containing regimens. Blood 2012;120:1954-7. [PubMed: 22936740] 


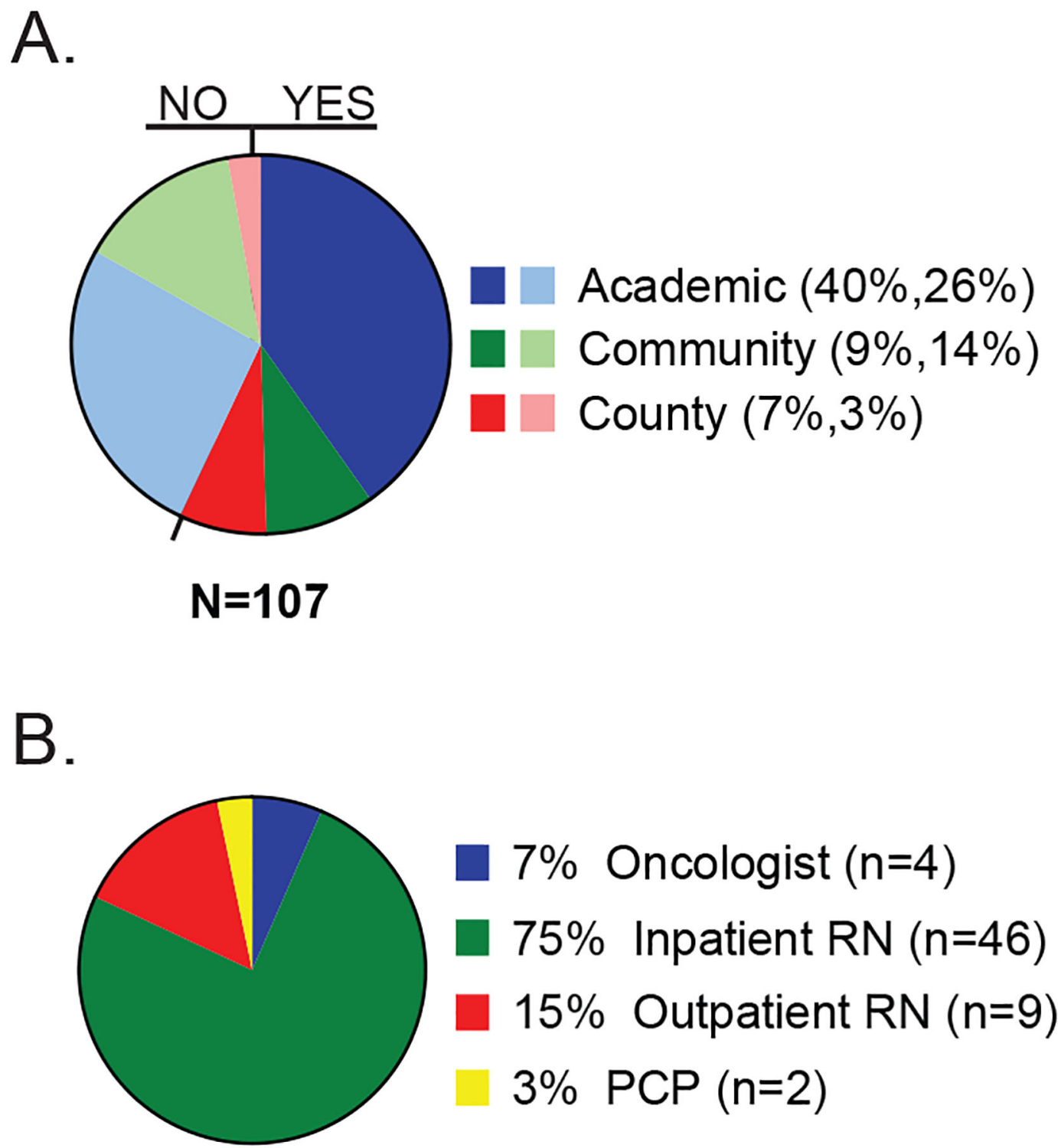

Figure 1. Documentation of influenza vaccination in patients with newly-diagnosed DLBCL. A) Percent of patients with documentation of influenza vaccination status among different hospitals. Light colors depict percent of patients without documented vaccination status. Parenthesis: percent of patients with vaccine documentation, percent of patients without vaccine documentation. B) Healthcare personnel documenting vaccination status. $n=$ actual number of patients. 


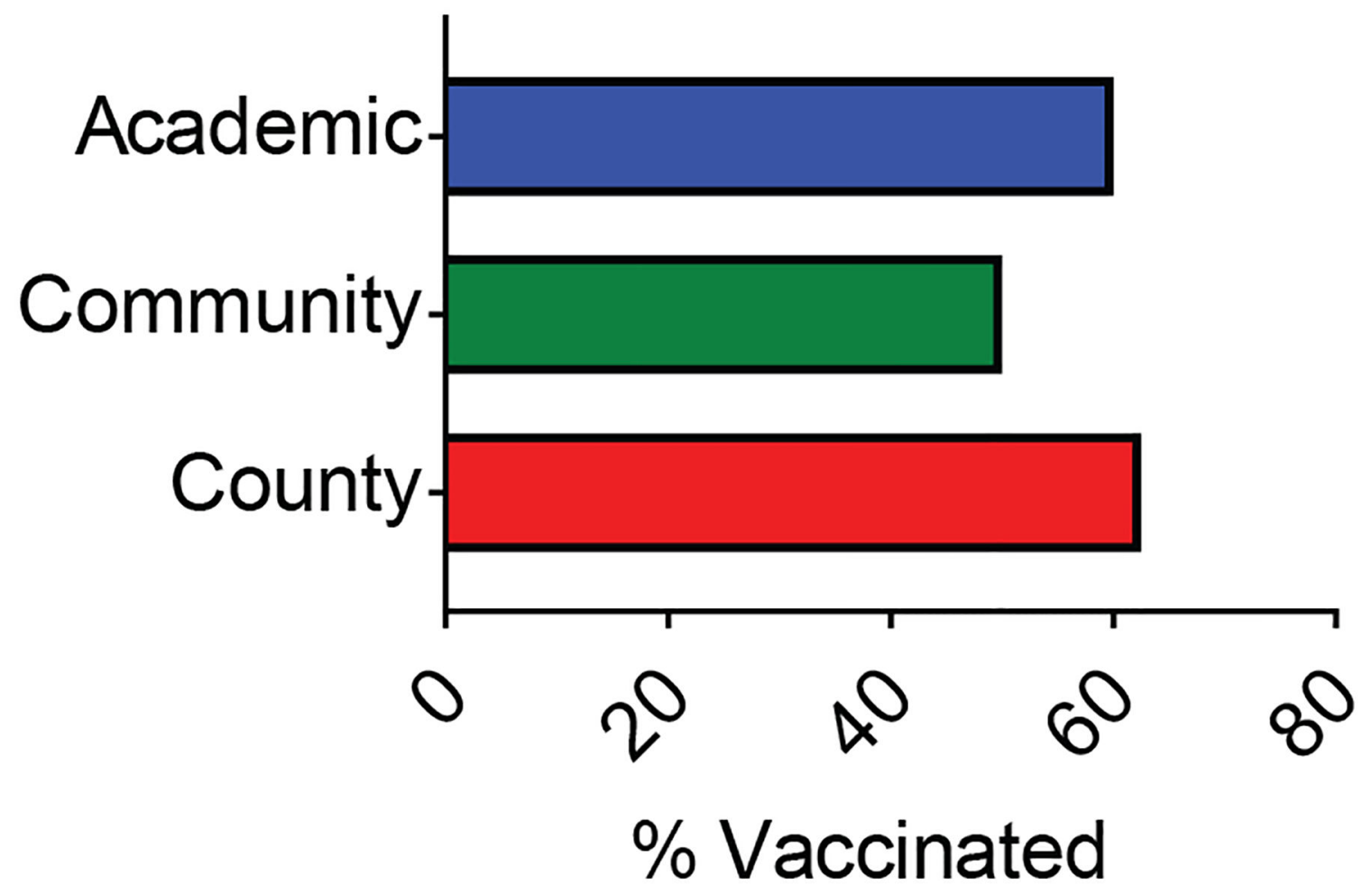

Figure 2. Influenza vaccination rates in patients with newly-diagnosed DLBCL among different hospitals.

Percent of patients who were vaccinated among different hospitals assessed. Influenza vaccination rate in this cohort was $57.4 \%$ (37 patients of 61$)$. 
Table 1.

\section{Patient Characteristics}

\begin{tabular}{lllll}
\hline & Total $(\mathbf{n = 1 0 7})$ & Academic $(\mathbf{n = 7 1})$ & Community $(\mathbf{n = 2 5})$ & County $(\mathbf{n = 1 1})$ \\
Median age in years at diagnosis (range) & $58.7(21.0-87.9)$ & $59.75(24.7-87.9)$ & $60.7(21.0-79.4)$ & $52.8(24.1-68.0)$ \\
65 years or older (\%) & 33.6 & 36.6 & 36.0 & 9.1 \\
Male gender (\%) & 53.3 & 52.1 & 56.0 & 54.5 \\
Non-Caucasians (\%) & 32.7 & 26.8 & 17.4 & 90.9 \\
Hispanics (\%) & 5.6 & 1.4 & 8.7 & 27.3 \\
Median number of visits during first influenza season & $7(1-29)$ & $7(1-29)$ & $6(1-17)$ & $7(2-14)$ \\
after diagnosis of DLBCL (range) & & & &
\end{tabular}


Table 2.

Influenza Vaccine documentation and administration rate in newly diagnosed DLBCL patients among 3 hospitals

\begin{tabular}{lrr}
\hline Hospital Setting & Documentation rate Mean \% (95\% C.I.) & Vaccination refusal rate Mean \% (95\% CI) \\
Academic & $60.6(49.2-71.9)$ & $41.9(30.2-55.0)$ \\
Community & $40.0(20.8-59.2)$ & $50.0(19.0-81.0)$ \\
County & $72.7(46.4-99.0)$ & $37.5(4.0-71.0)$ \\
Total & $57.0(47.6-66.4)$ & $42.6(30.2-55.0)$
\end{tabular}

